\title{
Blind Source Separation based on Whale Optimization Algorithm
}

\author{
Ding-li $\mathrm{CHU}^{1 *}$, Hong $\mathrm{CHEN}^{1}$, and Han-yi $\mathrm{CHEN}^{2}$ \\ ${ }^{1}$ National University of Defense Technology, Hefei, China \\ 273630 Brigade, Fuzhou, China
}

\begin{abstract}
Aiming at the problem of linear instantaneous aliasing in blind source separation, a new method of blind signal separation using whale optimization algorithm is proposed in this paper, which provides a new research idea and method for blind signal separation. The new method adopts the method of independent component analysis, optimizes the objective function by using the whale optimization algorithm, realizes the blind separation of instantaneous aliasing signals, and effectively avoids the problem of complex parameters and slow convergence rate of the particle swarm optimization algorithm. The simulation results show that the performance of whale optimization algorithm is better than that of particle swarm optimization for blind source separation, and it is effective for blind signal separation.
\end{abstract}

\section{Introduction}

Blind Signal Separation (BSS) is a new research field developed in the late 20th century, when there is little available information on the source signal and the transmission channel, and the components of the source signal are extracted or recovered from the observed mixed signal ${ }^{[1]}$. Blind signal separation is a powerful signal processing method with many potential applications, especially in wireless communications, medical analysis, image processing, speech recognition, blind signal separation has become one of the hot topics in the field of signal processing and artificial neural networks in the world ${ }^{[2]}$ and has important theoretical significance and value.

Most methods of blind processing are unsupervised learning methods that construct objective functions based on certain independence criteria. At present, there are stochastic gradient method ${ }^{[3]}$, natural gradient meth-od ${ }^{[4]}$ and FastICA algorithm ${ }^{[5]}$ for the method of calcu-lation of the objective function. As for the gradient algorithm needs to calculate the nonlinear activation function and the pseudo inverse matrix, the calculation is large, the robustness is poor, and the convergence needs to be improved ${ }^{[6]}$, FastICA sensitive to the initial value and other issues. Lui et al. proposed a particle swarm optimization(PSO) based blind separation algorithm in [7], which is not easy to fall into the local extremum, but the parameters are set too much, and the convergence rate is slower; the basic particle swarm optimization was improved in [2], and the convergence speed has been further improved, but it also increases the complexity of the algorithm. In this paper, we introduce a method to separate signals by the whale optimization.

\section{Description of BSS}

Assume $\mathbf{x}(t)$ is a linear combination of $n$ unknown statisti-cal independent source signal $\mathbf{s}(t)$, which is

$$
\mathbf{x}(t)=\mathbf{A} \mathbf{s}(t)
$$

where $\quad \mathbf{x}(t)=\left[\mathrm{x}_{1}(t), \mathrm{x}_{2}(t), \ldots, \mathrm{x}_{m}(t)\right]^{\mathrm{T}}, \mathbf{s}(t)=\left[\mathrm{s}_{1}(t), \mathrm{s}_{2}(t)\right.$, $\left.\ldots, \mathrm{s}_{n}(t)\right]^{\mathrm{T}}, \mathbf{A}$ is a $m \times n$ order unknown hybrid matrix. Blind separation is to use a certain algorithm to process the observed $\mathbf{x}(t)$ when $\mathbf{A}$ and $\mathbf{s}(t)$ are unknown, so that the separation matrix $\mathbf{W}$ of $m \times n$ order is obtained, so that the output signal

$$
\mathbf{y}(t)=\mathbf{W} \mathbf{x}(t)=\mathbf{W A s}(t)
$$

is an estimate of the source signal $\mathbf{s}(t)$.

In order to ensure the possibility of blind source se-paration, some assumptions are needed. Usually for ${ }^{[8]}$ :

1) Mixing matrix $\mathbf{A}$ is full column rank, the number of source signals is not greater than the number of observed signals. (which is $n \leq m$ ).

2) From a statistical point of view, the sources are statistically independent of each other. In practical app-lications, this assumption is reasonable.

3) At most one signal is a Gaussian distribution.

Since there is no prior information such as source signal and channel, the signal amplitude and arrangement order after blind separation is difficult to determine, which is called fuzziness. Although there is

Corresponding author: $1240699100 @ \mathrm{qq} . c 0 m$ 
some ambiguity, in most scientific researches and practices ${ }^{[10]}$, the ambiguity of blind source separation will not have much effect on the result.

According to the large number theorem, the sum of several independent random variables tends to the Gauss distribution. Therefore, if the observed signal is a linear combination of the source signal, the nonGauss property of the source signal is stronger than the non-Gauss characteristic of the observed signal. According to this principle, the mutual independence of the separated sig-nals can be determined by the non-Gauss judgment [11]. When the non-Gauss property of the separation signal is maximum, it can be considered to be separated.

\section{BSS based on the Whale Optimization Algorithm}

\subsection{Whale Optimization Algorithm}

In 2016, inspired by the predatory behavior of humpback whales, Mirjalili $S$ et al. ${ }^{[8]}$ proposed a natural inspired group intelligence algorithm called Whale Optimization Algorithm (WOA). Mathematical model of the algorithm includes three ways of enveloping prey, bubble-net attack and searching for predation. Each time the location is updated, each individual selects one of the updating methods according to certain criteria. Here are three ways to update.

\subsubsection{Encricling prey}

Since the search space of the optimal position is not known in advance, the WOA algorithm assumes that the current best candidate solution is the target prey position or the position closest to the target prey. After the best proxy is defined, other search agents will approach the best agent. The mathematical expressions for its location update are as follows

$$
\begin{aligned}
& \mathbf{X}(j+1)=\mathbf{X}(j)-A \cdot \mathbf{D} \\
& \mathbf{D}=\left|C \cdot \mathbf{X}^{*}(j)-\mathbf{X}(j)\right|
\end{aligned}
$$

where $A$ and $C$ are coefficient variables, $\mathbf{X}^{*}(j)$ is the best whale location so far, $j$ is the number of current itera-tions.

The calculation methods of coefficient variable $A$ and $C$ are as follows:

$$
\begin{gathered}
A=2 a \times r-a \\
C=2 r
\end{gathered}
$$

where $a$ is a constant, and its value decreases linearly from 2 to 0 , and the expression is $a=2-\frac{2 j}{M}$, where
$M$ is the maximum number of iterations; $r$ is a random number on $[0,1]$.

\subsubsection{Bubble-net attacking method}

In order to simulate the behavior of humpback puff behavior, a mathematical model is established as follows:

a)Shrinking encircling mechanism: This behavior is achieved by reducing the value of $a$ in equation (5). At the same time, $A$ 's range of change $[-a, a]$ also decreases as a decreases. When $A$ is in the range $[-1$, $1]$, the position of the next search agent for whales will be between the current position and the prey position. The arbitrary position, which simulates the behavior of humpbacks attacking prey, shows the ability to develop local search.

b)Spiral updating position: The mechanism first calculates the distance between the whales and the prey, and then establishes a spiral mathematical model between the whales and their prey to simulate the humpback whales' spiral swimming behavior:

$$
\begin{gathered}
\mathbf{X}(j+1)=\mathbf{D}^{\prime} \mathrm{e}^{b l} \cos (2 \pi l)+\mathbf{X}^{*}(j) \\
\mathbf{D}^{\prime}=\left|\mathbf{X}_{j}^{*}-\mathbf{X}_{j}\right|
\end{gathered}
$$

where $\mathbf{D}^{\prime}$ is the distance between the $i$ th whale to the best solution obtained so far; $b$ is a defined logarithmic spiral shape constant; and $l$ is a random number between $[-1,1]$.

In order to simulate this synchronized behavior, humpback whales not only spiral prey to prey, but also update the whale position with $50 \%$ probability as the selection threshold in updating the position of individual whales. The equation is as follows

$$
\mathbf{X}(j+1)= \begin{cases}\mathbf{X}(j)-A \cdot \mathbf{D} & p<0.5 \\ \mathbf{D}^{\prime} \mathrm{e}^{b l} \cos (2 \pi l)+\mathbf{X}(j) & p \geq 0.5\end{cases}
$$

where $l$ is a random number on $[0,1]$.

\subsubsection{Search for prey}

In addition to the bubble net search, humpback whales also randomly search prey. The change method based on the coefficient $A$ can also be used to search for prey. In fact, the whale group can search the prey randomly according to each other's position. Therefore, when $A$ is not in $[-1,1]$, random value is forced to force whales to deviate from prey, so as to search for other more suitable prey to enhance the search and predation ability of the algorithm, so that WOA has better global search performance. The equation is as follows

$$
\begin{gathered}
\mathbf{D}=\left|C \cdot X_{\text {rand }}-X(j)\right| \\
\mathbf{X}(j+1)=\mathbf{X}_{\text {rand }}-A \cdot \mathbf{D}
\end{gathered}
$$

where $\mathbf{X}_{\text {rand }}$ is the location of a random individual in the current whale group. 


\subsection{Principle of separation}

In order to blindly separate the mixed signal $\mathbf{x}(t)$, we propose to use the WOA algorithm to solve the separation matrix $\mathbf{W}$ in the equation (2). In order to obtain the separation matrix $\mathbf{W}$, we need a feasible independence criterion between random variables, and then obtain the corresponding blind source separation algorithm by optimizing the separation criterion expression. The components of the estimated signal are independent of each other. Common discriminant criteria are: minimum mutual information criterion, maximum information criterion, non-Gaussian maximization criterion and so on. In order to verify the performance of the algorithm, the objective function of [2] is adopted here. The independence criterion given in [2] is as follows:

The components $\mathrm{y}_{i}$ and $\mathrm{y}_{j}$ are independent of each other meaning that for any measurable function $g_{1}(\cdot)$ and $g_{2}(\cdot)$, then

$$
E\left[g_{1}\left(y_{i}\right) g_{2}\left(y_{j}\right)\right]-E\left[g_{1}\left(y_{i}\right)\right] E\left[g_{2}\left(y_{j}\right)\right]=0, \forall i \neq j
$$

that is, $g_{1}(\cdot)$ and $g_{2}(\cdot)$ are irrelevant to each other. Therefore, in order to judge whether the signals $\mathrm{y}_{i}$ and $\mathrm{y}_{j}$ are independent from each other, it is theoretically necessary to traverse the correlations of $g_{1}(\cdot)$ and $g_{2}(\cdot)$, but this is not practical and not necessary. In fact, the second and higher order correlation coefficients for signals $\mathrm{y}_{i}$ and $\mathrm{y}_{j}$ can be defined as follows:

$$
\begin{gathered}
c_{s}\left(y_{i}(t), y_{j}(t)\right)=\frac{\operatorname{cov}\left[y_{i}(t), y_{j}(t)\right]}{\sqrt{\operatorname{cov}\left[y_{i}(t)\right] \operatorname{cov}\left[y_{j}(t)\right]}} \\
c_{h}\left(\varphi_{i}\left(y_{i}(t)\right), y_{j}(t)\right)=\frac{\operatorname{cov}\left[\varphi_{i}\left(y_{i}(t)\right), y_{j}(t)\right]}{\sqrt{\operatorname{cov}\left[\varphi_{i}\left(y_{i}(t)\right)\right] \operatorname{cov}\left[y_{j}(t)\right]}}(
\end{gathered}
$$

where $\operatorname{cov}[x(t), y(t)]=E\left\{\left[x(t)-m_{x}\right]\left[y(t)-m_{y}\right]\right\}, \operatorname{cov}$ $[x(t)]=E\left\{\left[x(t)-m_{x}\right]^{2}\right\}, m_{x}=E[x(t)]$ and $\varphi_{i}\left(y_{i}(t)\right)$ is a nonlinear function of $y_{i}$.

The independence of the two signals can be measured by the second-order correlation coefficient and the higher-order correlation coefficient. The guideline is: If $\left[c_{s}\left(y_{i}(t), y_{j}(t)\right)\right]^{2}+\left[c_{h}\left(\varphi_{i}\left(y_{i}(t)\right), y_{j}(t)\right)\right]^{2}+\left[c_{h}\left(y_{i}(t)\right.\right.$, $\left.\varphi_{j}\left(y_{j}(t)\right)\right]^{2}=0$, the two signals $\mathrm{y}_{i}$ and $\mathrm{y}_{j}$ are independent of each other.

Therefore, the following objective function can be used as the measure of mutual independence between the various components of $\mathbf{Y}(t)$ :

$$
J(\mathbf{W})=\sum_{i=1}^{n} \sum_{j=1, j \neq i}^{n}\left\{\begin{array}{l}
{\left[c_{s}\left(y_{i}(t), y_{j}(t)\right)\right]^{2}+} \\
{\left[c_{h}\left(\varphi_{i}\left(y_{i}(t)\right), y_{j}(t)\right)\right]^{2}+} \\
{\left[c_{h}\left(y_{i}(t), \varphi_{j}\left(y_{j}(t)\right)\right]^{2}\right.}
\end{array}\right\}
$$

For the non-linear function $\varphi_{i}(\cdot)$ in the objective function (15), the nonlinear function used in [14] is selected, which is as follows

$$
\varphi_{i}\left(y_{i}\right)=\alpha_{i}\left(\kappa_{3}^{i}, \kappa_{4}^{i}\right) y_{i}^{2}+\beta_{i}\left(\kappa_{3}^{i}, \kappa_{4}^{i}\right) y_{i}^{3}
$$

where $\kappa_{3}^{i}=E\left[y_{i}^{3}\right]$ and $\kappa_{4}^{i}=E\left[y_{i}^{4}\right]-3$ represent the skewness and kurtosis of $y_{i}$, respectively. And $\alpha_{i}\left(\kappa_{3}^{i}, \kappa_{4}^{i}\right)=-\frac{1}{2} \kappa_{3}^{i}+\frac{9}{4} \kappa_{3}^{i} \kappa_{4}^{i}, \quad \beta_{i}\left(\kappa_{3}^{i}, \kappa_{4}^{i}\right)=-\frac{1}{6} \kappa_{4}^{i}+\frac{3}{2}\left(\kappa_{3}^{i}\right)^{2}$ $+\frac{3}{4}\left(\kappa_{4}^{i}\right)^{2}$

Here we use the whale optimization algorithm to optimize the objective function (15).

\subsection{Algorithm Steps}

The steps of blind source separation based on the whale optimization algorithm are as follows:

1) Collect and sample the observed signals, centralize and pre-whiten them.

2) WOA algorithm parameters initialization. Taking the elements of the separation matrix $\mathbf{W}$ as the location information $\mathbf{X}=\left[X_{1}, X_{2}, X_{3}, \ldots, X_{n}\right]$ of the whale individual. The location of whale iteration is initialized randomly, and WOA parameters are set up, including population number $N$, maximum iteration number $M$, logarithmic spiral shape constant $b$, random number $l$, current iteration number $j$ and algorithm termination condition.

3) Calculate individual fitness by the equation (14) ,then find and save the best whale individual position $\mathbf{X}^{*}$ in the group.

4) If $j \leq M$,update $a 、 A 、 C 、 l$ and $p$.

5) When $p<0.5$, if $A<1$, update the space position of the current whale individual by the equation (3);if $A \geq 1$, the whale individual location $\mathbf{X}_{\text {rand }}$ is randomly selected from the current group, and update the space location of the current whale individual by the equation (11).

6) When $p \geq 0.5$, Update the space location of the current whale individual by the equation (7).

7) Calculate population fitness by the equation (15), then find and save the best whale individual position $\mathbf{X}^{*}$ in the group. Determine whether the algorithm meets the termination condition, if satisfied, go to step 8; otherwise, let $j=j+1$, repeat steps $4 \sim 7$.

8) Output optimum individual whale fitness value and its spatial location $\mathbf{X}^{*}$, which is the element of the best separation matrix $\mathbf{W}^{*}$.

9) According to the obtained separation matrix $\mathbf{W}^{*}$, estimate the source signal by the equation(2).

\section{Simulation\&Verification}

This section is based on the Matlab2014a platform simulation to verify and analyze the blind signal separation performance based on WOA. 


\section{Experiment}

Two-way simulation signals are used as the source signal, and the sampling frequencies are 1024 and 512, respectively, and a total of 5000 sampling points are used. Two-way road simulation signal is as follows

$$
\begin{aligned}
s 1= & 30 * \sin \left(1 * \pi * N / f s_{1}\right)+0.2 * w g n(1, N, 1) ; \\
s 2= & 0.5 * \sin \left(2 * \pi * N / f s_{2}\right)+0.5 * \cos \left(4 * \pi * N / f s_{2}\right) \\
& +0.2 * w g n(1, N, 1) ;
\end{aligned}
$$

In order to verify the performance of the algorithm, experiment setup is the same as [2], and the mixed matrix is as follows

$$
\mathbf{A}=\left[\begin{array}{cc}
1 & 1.5 \\
1.4 & 1
\end{array}\right]
$$

In the process of experiment, the number of whale population is 20 and the number of iterations is 50 . Compared with [2], the population number of IPSO algorithm is 20 , the number of iterations is 50, and two learning factors are 0.8 , then the simulation results are as follows:
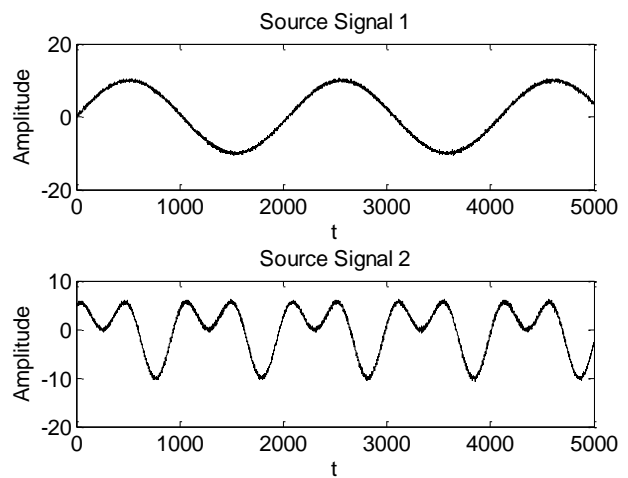

Fig.1. Source Signals
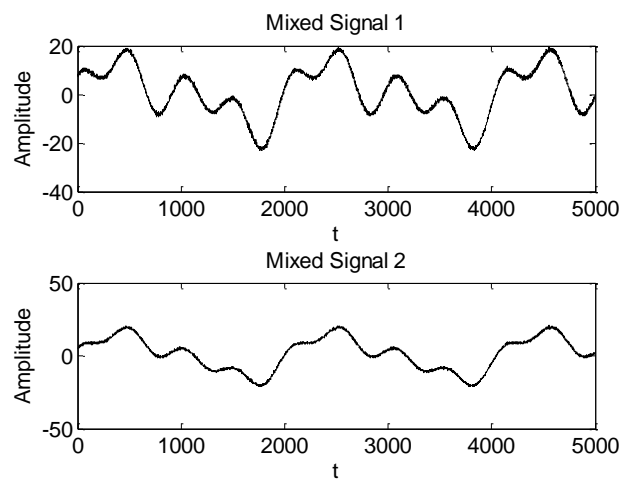

Fig.2. Mixed Signals
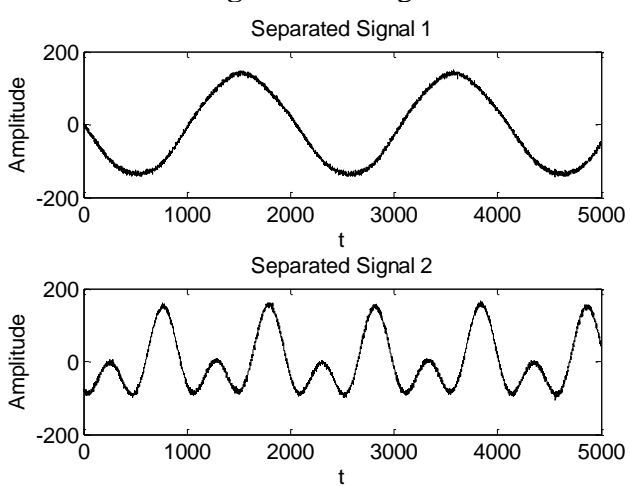

Fig.3. Separated Signals(WOA)
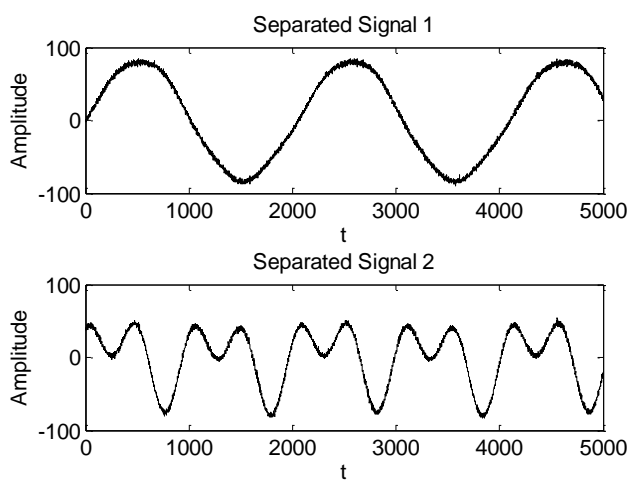

Fig.4. Separated Signals(IPSO)

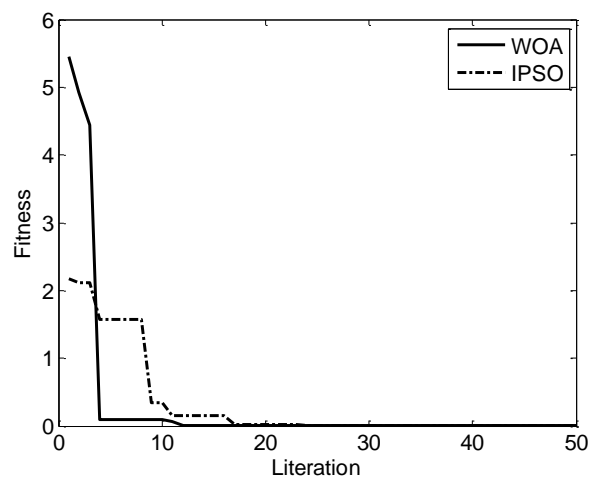

Fig.5. Algorithm convergence curve

As can be seen from Figure 1-4, there is uncertainty in the magnitude and order of the separation signals, but this does not affect the separation effect. It can be seen from the comparison chart of the convergence curve in Figure 5 that after 12 iterations, the fitness of the whale algorithm has converged, and the convergence rate shows that the convergence rate is very fast; and the particle swarm algorithm converges at about 20 steps and the convergence speed is slower than that of the whale algorithm. WOA shows good performance in convergence speed in BSS.

In order to quantitatively evaluate the separation effect, the similarity coefficient matrix $\zeta$ between the separation signal and the source signal is used as an inspection index. After simulation calculations, the similarity matrix based on WOA is $\zeta_{1}=\left[\begin{array}{ll}0.9997 & 0.0462 \\ 0.0138 & 0.9994\end{array}\right]$, and the similarity matrix based on IPSO is $\zeta_{2}=\left[\begin{array}{ll}0.9847 & 0.0704 \\ 0.0408 & 0.9891\end{array}\right]$, we can see that the precision of WOA algorithm is higher than that of IPSO algorithm.

\section{Conclusion}

In the complex channel environment, the separation of multiple signals has important practical significance and research prospects. Whale optimization algorithm is a new evolutionary computation method, which is inspired by the study of predatory behavior of whales. The whale optimization algorithm was introduced into the blind signal separation. The source signal was successfully separated through simulation experiments. Compared 
with the improved particle swarm algorithm, the whale optimization algorithm has certain advantages in separation accuracy and convergence speed, and the convergence speed is high while the precision is high. It is also faster and can provide a new research idea and method for blind signal separation.

\section{References}

1. MAO Xin, XU Kai, LIU Jie. Present Situation and Development of Blind Signal Separation Technology[J]. Communications Technology. 2013(8):24-26.

2. Gao Y, Liu H L. An improved particle swarm optimizer and its application in blind signals separation[J]. Journal of Guangzhou University, 2011.

3. Lee T W, Girolami M, Sejnowski T J. Independent component analysis using an extended infomax algorithm for mixed subgaussian and supergaussian sources[J]. Neural Computation, 1999, 11(2):417.

4. Amari S. Natural Gradient Works Efficiently in Learning[J]. Neural Computation, 2014, 10(2):251276.

5. Hyv\&xE, Rinen A. Fast and robust fixed-point algorithms for independent component analysis[J]. IEEE Transactions on Neural Networks, 1999, 10(3):626.

6. Cichocki A, Amari S I, Cao J. Neural Network Models for Blind Separation of Time Delayed and Convolved Signals[J]. Ieice Transactions on Fundamentals of Electronics Communications \& Computer Sciences, 1997, 80(9):1595-1603.

7. LIU Hui, LI Bai. Application of Particle Swarm Algorithm in Blind Source Separation[J]. Modern Electronics Technique, 2010, 33(17):94-96.

8. Mirjalili S, Lewis A. The Whale Optimization Algorithm[J]. Advances in Engineering Software, 2016, 95:51-67.

9. ZHENG Hui. Theory and Technology on Blind Signal Processing of Communication. National Defense Industry Press, 2013.

10. ZHANG Wen-ai, LIU Jun-hao. Blind Source Separation Based on Particle Swarm Optimization[J]. Journal of Taiyuan University of Technology, 2006, 37(2):169-172.

11. MA Jian-cang, NIU Yi-long, CHEN Hai-yang. Blind Signal Processing[M]. National Defense Industry Press, 2006.

12. WANG Meng, ZHANG Wen-ai. BSS Algorithm Based on APSO Application in Image Processing[J]. Video Engineering,2014,38 (19):32-35.

13. LIU Jun-hao. Research on Blind Source Separaton Based on Particle Swarm Optimization and Fish Swarm Algorithm[D]. Taiyuan University of Technology, 2006.

14. LU Gang, TAN De-jian. Improved on regulating definition of antibody density of immune
algorithm[C]. Proceedings of the 9th international conference on neural information processing(ICONIP'02),2002,5:2669-2672. 